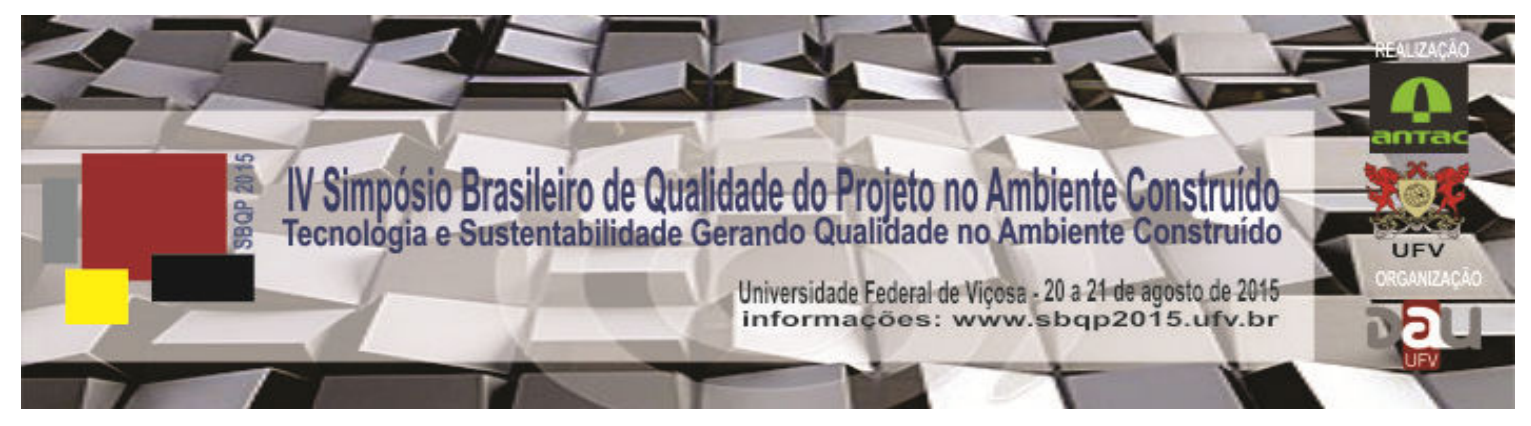

\title{
BARREIRAS FÍSICAS X BARREIRAS BIOLÓGICAS: A QUALIDADE DO ESPAÇO URBANO E AS QUEDAS DE IDOSOS1
}

\author{
FONSECA, Talita da C. de Oliveira \\ Universidade Federal de Viçosa, e-mail: talitaufv@yahoo.com.br \\ CARVALHO, Aline Werneck Barbosa \\ Universidade Federal de Viçosa, e-mail: alinewbc@gmail.com \\ TIBÚRCIO, Túlio Márcio de Salles \\ Universidade Federal de Viçosa, e-mail: tmst83@hotmail.com
}

\begin{abstract}
RESUMO
Os ambientes construídos, dentre eles os espaços urbanos, devem ser adequados às necessidades dos idosos de modo a evitar o risco de quedas. A identificação de fatores de riscos ambientais causadores de queda é notável no sentido de se estabelecer estratégias para a sua prevenção. O presente trabalho é parte de pesquisa de mestrado em andamento e busca analisar a relação entre as barreiras físicas do espaço urbano contíguo à Unidades Básicas de Saúde (UBSs) e as alterações funcionais do envelhecimento no que se refere às quedas de idosos. Nesta etapa, foram empregados os seguintes métodos: revisão de literatura para fundamentação da pesquisa e para possibilitar o levantamento do estado da arte, pesquisa documental nos acervos dos órgãos responsáveis pelas UBSs de uma cidade de médio porte, onde foram coletados dados e registros a fim de caracterizá-las quanto à sua área de abrangência e entrevistas exploratórias. Como resultado desta parte da pesquisa, as UBSs foram estratificadas quanto à sede própria ou alugada, onde foram selecionadas as UBSs de sede própria. Destas, foram destacados o número de pessoas cadastradas, o total de idosos e a porcentagem de idosos em relação à população cadastrada. Estes resultados contribuirão para a segunda parte da pesquisa que irá caracterizar o espaço urbano contíguo à UBS em estudo quanto às barreiras físicas; identificar as alterações funcionais decorrentes do processo de envelhecimento e correlacioná-las com o uso do espaço; verificar como os idosos percebem e se comportam no espaço urbano contíguo à UBS e descrever a mobilidade dos idosos no espaço urbano adjacente à UBS. A pesquisa sobre os fatores de risco para quedas de idosos no ambiente extradomiciliar justifica-se uma vez que vários estudos vêm sendo realizados com idosos institucionalizados ou no âmbito doméstico e poucos têm focado a sua relação com o espaço urbano.
\end{abstract}

Palavras-chave: Barreiras Físicas, Idoso, Acessibilidade, Quedas.

\begin{abstract}
The built environments, including urban spaces should be suitable to the needs of the elderly in order to avoid the risk of falls. The identification of environmental risk factors
\end{abstract}

\footnotetext{
1 Trabalho apresentado no IV SBQP 2015. Universidade Federal de Viçosa. Disponível em: doi> http://dx.doi.org/10.18540/2176-4549.6045
} 
that generate falls is remarkable in the sense of establishing strategies for their prevention. This work is part of master's research in progress and seeks to analyze the relationship between the physical barriers of the urban space adjacent to the Basic Health Units (Unidades Básicas de Saúde - UBSs) and aging functional changes in relation to falls in the elderly. At this stage, it was employed the following methods: literature review to enable the state of the art, documental research in the records of the UBS responsible agencies in a medium-sized city, where data were collected in order to characterize them within their coverage area, and exploratory interviews. As a result of the research, the UBS were stratified as to own or rented head office, being selected the UBS which has its own space. From those, the number of people registered was highlighted and the total number of elderly and the percentage of older people relative to the population were registered. These results will contribute to the second part of the research that will characterize the urban space adjacent to the studied UBS related to the physical barriers; to identify the functional alterations caused by the aging process and to correlate them with the use of space; to check how the elderly perceive and behave in urban areas adjacent to UBS and to describe elderly mobility in urban areas adjacent to UBS. Research on the risk factors for elderly falls in extradomiciliar environment is justified since several studies have been conducted with institutionalized elderly or in the domestic environment and few have focused their relationship with the urban space.

Keywords: Physical Barriers, Elderly, Accessibility, Falls.

\section{INTRODUÇÃO}

O crescimento da população idosa é um fenômeno mundial, porém no Brasil, essa transição demográfica está ocorrendo de forma radical e acelerada. As projeções indicam que em 2020 o Brasil será o sexto país do mundo em número de idosos. No entanto, o prolongamento da vida só pode ser considerado como uma real conquista na medida em que se agregue qualidade aos anos adicionais de vida (VERAS, 2009). Biologicamente, o envelhecimento é um processo natural, dinâmico, progressivo e irreversível, que se instala em cada indivíduo desde o nascimento e o acompanha por todo o tempo de vida, culminando com a sua morte. Nesse trajeto provoca no organismo inúmeras modificações morfológicas, fisiológicas e bioquímicas (MARTINS, 2006).

No Brasil, considera-se idoso a pessoa com idade igual ou superior a 60 anos (BRASIL, 2003). No entanto, é necessário reconhecer os limites dessa definição, uma vez que, podem ocorrer variações entre as idades cronológica e fisiológica de um indivíduo.

Mesmo diante da dificuldade em definir quem é o idoso, é evidente a diminuição da sua capacidade funcional. Entende-se por capacidade funcional a habilidade física e mental para manter uma vida independente e autônoma, e a possibilidade da plena realização de uma tarefa ou ação pelo indivíduo (MARCHON; CORDEIRO; NAKANO, 2010). De acordo com Cunha e Costa (2011), algumas alterações funcionais próprias do envelhecimento influenciam diretamente no uso do espaço. Com relação ao sistema musculoesquelético, por exemplo, há diminuição da agilidade, da coordenação, do equilíbrio e da flexibilidade. Essas alterações prejudicam o equilíbrio do idoso, causando mudanças na postura e na mobilidade. Já no sistema cognitivo, destacam-se a diminuição da audição e as mudanças fisiológicas do processo visual. No estudo, as alterações funcionais decorrentes 
do processo de envelhecimento serão denominadas como barreiras biológicas.

Em decorrência do processo de envelhecimento, a queda, considerada como um evento não intencional que tem como resultado a mudança de posição do indivíduo para um nível mais baixo, tem se tornado uma ocorrência frequente e um problema crescente entre os idosos (NICOLUSSI et al., 2012). Esses episódios podem estar relacionados a processos intrínsecos ao envelhecimento como, por exemplo, a redução da acuidade visual e diminuição de força muscular, ou mesmo a fatores extrínsecos ligados aos riscos ambientais somados aos fatores comportamentais, relacionados com o uso e percepção do espaço por parte do idoso frente à demanda imposta pelo ambiente e sua capacidade funcional real (MESSIAS; NEVES, 2009).

Mesmo tendo o quadro de envelhecimento comprovado no Brasil, isso não significa que o país e suas cidades estejam preparados para lidar com este fenômeno, suas consequências e impactos na vida social e familiar. Para Liboreiro et al. (2004), o prolongamento da vida determina a necessidade de as sociedades reestabelecerem um espaço digno para a existência de pessoas idosas.

No presente estudo, serão adotadas algumas definições de acordo com a legislação vigente e estas serão aplicadas no que se refere às quedas. Por barreira, entende-se "qualquer entrave ou obstáculo que limite ou impeça o acesso, a liberdade de movimento e a circulação com segurança das pessoas". Tais barreiras podem ser classificadas como arquitetônicas urbanísticas, que são aquelas existentes nas vias públicas e nos espaços de uso público; e as arquitetônicas nos transportes, sendo aquelas existentes nos meios de transportes. Por barreira ambiental, deve-se entender qualquer elemento natural que impeça a aproximação, transferência ou circulação no espaço urbano (ABNT, 2000).

Quanto mais um ambiente se ajusta às necessidades do usuário, mais confortável e seguro ele é. Todavia, se ocorre o inverso, quando o ambiente construído não leva em conta as necessidades ou limitações humanas, ele pode chegar a ser mais inóspito que o meio natural (CAMBIAGHI, 2007).

A Psicologia Ambiental, como uma subárea da Psicologia, estuda as interrelações do homem com o meio ambiente: analisa percepções, atitudes e comportamentos individuais ou coletivos em explícita relação aos contextos físico e social dentro dos quais se vive (GUNTHER; PINHEIRO; GUZZO, 2006). Dessa forma, a criação do espaço arquitetônico deve se preocupar com o usuário, com sua percepção e sua maneira de ver. O espaço deve favorecer o comportamento harmonioso do homem, mais do que atender às suas necessidades básicas (OKAMOTO, 2002). De acordo com Cambiaghi (2007), para um ambiente urbano alcançar o status de agradável e cômodo, este precisa possibilitar a chegada a todos os lugares, possibilitar a entrada em todos os edifícios e possibilitar a utilização de todas as instalações públicas e privadas e dos espaços externos em que elas se inserem.

Outro fator relacionado com o processo de envelhecimento consiste em que, diferentemente das pessoas dos demais grupos etários, os idosos procuram os serviços de saúde com maior frequência. Para chegar até estes serviços, é necessário observar as condições de mobilidade e acessibilidade urbana. 
Portanto, deslocar-se, quando se deseja ou necessita, significa poder usufruir dos bens e serviços de uma cidade (BARRETO, 2012).

Como parte da Atenção Primária à saúde (AP), a Unidade Básica de Saúde (UBS) representa o primeiro contato da população com o serviço de saúde do município. Neste caso, primeiro contato significa acesso e utilização do serviço de saúde para cada novo evento de saúde ou novo episódio de um mesmo evento. Para que funcione como primeiro recurso, este serviço deve ser de fácil acesso e disponível (CONASS, 2011).

Devido à importância das UBSs para a AP, tornam-se necessários métodos de planejamento ou de adequação do espaço urbano onde estas estão inseridas, considerando a diversidade de usuários e as particularidades do público idoso, pois a prestação de cuidados reclama estruturas que permitam a este público usufruir integralmente dos serviços oferecidos pelas cidades. A importância de se identificar os fatores de risco para quedas de idosos no ambiente extradomiciliar se dá uma vez que vários estudos vêm sendo realizados com idosos institucionalizados ou no âmbito doméstico e poucos têm focado a sua relação com o espaço urbano, que muitas vezes não é acompanhado por uma infraestrutura eficiente.

É fundamental que estes espaços sejam projetados pensando-se e garantindose a segurança dos idosos, de maneira que o ambiente físico possa aprimorar, manter ou recuperar a sua capacidade funcional. Na medida em que se tornam necessárias intervenções nas edificações frequentadas pelos idosos, a fim de se reduzir a probabilidade de quedas, é preciso também redirecionar as práticas no que se refere à adequação do espaço urbano, considerado como fundamental para a manutenção da autonomia e independência dos mesmos.

O estudo das questões qualitativas relacionadas a tais espaços no que se refere aos riscos de quedas serve de subsídio para a elaboração de projetos arquitetônicos, para formuladores de políticas públicas e para gestores de saúde comprometidos com a qualidade de vida dos idosos.

\section{OBJETIVOS}

O objetivo principal da pesquisa é analisar a relação entre as barreiras físicas do espaço urbano contíguo às UBSs e as alterações funcionais do envelhecimento no que se refere às quedas de idosos. Outros objetivos específicos foram traçados para o desenvolvimento da pesquisa sendo eles: caracterizar as UBS na cidade de Viçosa-MG quanto à área de abrangência, número de famílias, número de idosos cadastrados e serviços prestados; caracterizar o espaço urbano contíguo à UBS quanto às barreiras físicas; identificar as principais alterações funcionais decorrentes do processo de envelhecimento relacionadas com os principais agravantes para risco de quedas e correlacioná-los com o uso do espaço; verificar como os idosos percebem e se comportam no espaço urbano contíguo à UBS em estudo e descrever a mobilidade dos idosos no espaço contíguo à UBS, considerando os modos de transporte utilizados.

\section{METODOLOGIA}


O presente trabalho é parte de pesquisa de mestrado do Departamento de Arquitetura e Urbanismo da Universidade Federal de Viçosa, em andamento, e insere-se na linha de pesquisa Comportamento Ambiental do Espaço Construído que compõe a área de concentração Planejamento e Avaliação do Espaço Construído.

A metodologia utilizada neste trabalho consiste em revisão de literatura que incluiu tópicos relacionados ao envelhecimento, barreiras físicas, barreiras biológicas e quedas dos idosos, a fim de entender o estado da arte, que serviu como embasamento da pesquisa. Além disso, foi realizada uma pesquisa documental nos acervos dos órgãos responsáveis pelas UBSs de uma cidade de médio porte, para definição de estudos de casos onde foram coletados dados e registros a fim de caracterizá-las quanto à sua área de abrangência. Para possibilitar maior familiaridade com o objeto de estudo, foram realizadas entrevistas exploratórias com as enfermeiras responsáveis técnicas pela UBS e com os Agentes Comunitários de Saúde. A entrevista exploratória ou informal (GIL, 1999) tem por finalidade encontrar pistas de reflexão, ideias e hipóteses de trabalho; permite ganho de tempo e economia de meios. O seu objetivo está ligado aos objetivos da investigação e é sempre pedida pelo investigador e não pelo interlocutor (QUIVY, CAMPENHOUDI, 2005). Com o andamento da pesquisa serão utilizados outros métodos como Walkthrough, Walkthrough Comentada, Entrevistas e Observação Comportamental, muito utilizados em Avaliação Pós-Ocupação. Estes métodos constituirão a segunda etapa da pesquisa, cujos instrumentos de coleta de dados já foram elaborados, mas não fazem parte do escopo deste artigo.

\section{REVISÃO DE LITERATURA}

Mais do que qualquer época, o século 20 caracterizou-se por profundas transformações, destacando-se no seu transcurso o aumento do tempo de vida da população. Diante desta realidade, cada vez mais o tema do envelhecimento vem sendo abordado, tanto nos países desenvolvidos quanto nos países do Terceiro Mundo. No Brasil, o envelhecimento da população é um fenômeno relativamente recente e os estudos sobre o tema não são numerosos. Entretanto, as pesquisas brasileiras têm apontado que o processo de envelhecimento da população brasileira é considerado irreversível, enfatizando a importância dos estudos voltados a este público (ARAÚJO; ALVES, 2000).

Apesar do evidente crescimento da população idosa e das transformações sociais dele decorrentes, a discussão sobre o envelhecimento se dá num contexto em que a diversidade de conceitos para explicar quem é o idoso e como se caracteriza o processo de envelhecer ainda está longe de diminuir. Assim, no Brasil e no mundo, o critério etário é o mais utilizado, embora não atenda à complexidade constituinte desse processo (TORRES; SÁ, 2008).

Além disso, o envelhecimento não é um processo homogêneo. Cada pessoa vivencia essa fase da vida de uma forma, considerando sua história particular e todos os aspectos estruturais a eles relacionados com saúde, educação e condições econômicas. O perfil dos idosos também é outro, porque os mesmos estão construindo uma identidade própria, que inclui a luta pela manutenção da autoestima, com posturas ativas, busca pela melhoria nas 
questões relacionadas à saúde e qualidade de vida, entre outros (BALBINOTTI, 2003).

De acordo com Bins Ely e Dornelles (2006), os idosos são usuários complexos, pois cada modificação fisiológica pode acarretar uma limitação diferente no uso do espaço e dos equipamentos. Por outro lado, entre os idosos há muitas pessoas ativas, que são responsáveis por várias atividades de vida realizadas em espaços urbanos. Para Messias e Neves (2009), no Brasil aproximadamente $30 \%$ das pessoas com 65 anos e mais, que moram em comunidades, ou seja, que não foram institucionalizadas, tem um evento de queda a cada ano e metade dos idosos que caem repetem o evento.

As quedas na população idosa resultam em uma série de consequências negativas, incluindo o declínio funcional, redução da qualidade de vida, morbidade, mortalidade e risco de hospitalização prolongada. Sendo assim, a redução dos riscos de quedas tornou-se uma prioridade internacional (CHILD et al., 2012).

Entre outros problemas trazidos pelo crescimento demográfico, encontra-se a carência dos espaços urbanos em relação às barreiras físicas impostas aos idosos. Se no processo de concepção dos projetos não for considerada a diversidade de usuários, é possível que apenas uma porcentagem reduzida da população possa utilizar tais espaços confortavelmente e com segurança (CAMBIAGHI, 2007). Sendo assim, é importante reconhecer que a eliminação de barreiras físicas no ambiente frequentado pelo idoso depende de diferentes etapas de atuação - projeto, execução e fiscalização - e que o conjunto dessas ações pressupõe a integração de profissionais e equipes diversas (DISCHINGER; BINS ELY; PIARDI, 2012).

No âmbito da Psicologia Ambiental, a preocupação com o planejamento de ambientes construídos aparece com ênfase na busca de compatibilidade entre as características das edificações e os fins a que elas seriam dedicadas. Ainda que esta disciplina tenha nascido com a dupla vinculação ao estudo de problemas de degradação ambiental e elaboração de projetos para ambientes construídos, essa subárea da Psicologia evoluiu para abarcar muitos outros tipos de problemas no âmbito do fazer humano. Dentre os exemplos de objeto de estudo e intervenção estão: planejamento e avaliação de equipamentos urbanos, planejamento e intervenção no tecido urbano e os impactos de diferentes tipos de ambientes sobre populações específicas (GUNTHER; PINHEIRO; GUZZO, 2006).

No que se refere à saúde do idoso, as ações devem estimular a inter-relação e - convívio social, o respeito à autonomia e à independência e o fortalecimento dos laços familiares, numa perspectiva de prevenção ao asilamento (MINAS GERAIS, 2006). A queda, que é um conhecido fator de risco para uma futura incapacidade em indivíduos com 75 anos e mais, pode também resultar em maiores taxas de idosos residindo em Instituições de Longa Permanência (ILP), além da perda de confiança levando a uma restrição voluntária de atividade (CHOI; HAYWARD; LANGA, 2013). No Brasil, de acordo com a Política Nacional de Atenção Básica (PNAB), a AP é desenvolvida com o mais alto grau de descentralização, ocorrendo no local mais próximo da vida das pessoas. Ela deve ser o contato preferencial dos usuários, a principal porta de entrada e o centro de comunicação com toda a Rede de Atenção à Saúde. Por isso, é fundamental que ela se oriente pelos 
princípios da universalidade, da acessibilidade, da integralidade da atenção, entre outros (BRASIL, 2012).

A UBS é a unidade para realização de atendimentos de atenção básica e integral a uma população, de forma programada ou não, nas especialidades básicas, podendo oferecer assistência odontológica e de outros profissionais de nível superior. A assistência deve ser permanente e prestada por médico generalista ou especialista nestas áreas. A definição da área de atuação de uma equipe deve ser realizada, se possível, de forma conjunta pela população, por técnicos da Secretaria Municipal de Saúde e pela própria equipe. O cadastramento da população residente no território é necessário e o mesmo é realizado pelos ACSs, mesmo que uma parcela dela não utilize a unidade de saúde (CONASS, 2011).

Portanto, coloca-se em discussão se o local escolhido para a instalação das UBSs promove realmente a proximidade com os usuários e se o espaço urbano permite de fato seu uso, respeitando as capacidades e necessidades de todos seus usuários. Já que surgem novas oportunidades na medicina e no sistema público de saúde, que estão contribuindo para a qualidade e manutenção da estrutura física e emocional dos sujeitos, os olhares atuais devem observar também questões qualitativas, de formação de novas oportunidades e de políticas que promovam e proporcionem o envelhecer com qualidade (WOLFF, 2009). Em especial, deve-se levar em consideração as relações dos idosos com o espaço.

\section{RESULTADOS PRELIMINARES}

O estudo está sendo realizado em Viçosa-MG, município da região da Zona da Mata Mineira. A revisão de literatura permitiu o entendimento e contextualização do problema da pesquisa, principalmente o entendimento e identificação das barreiras biológicas caracterizadas pelas questões relacionadas às quedas dos idosos. Foram levantadas variáveis para o estudo que serão base das análises, apresentadas no Quadro 1.

As entrevistas exploratórias não estruturadas com os agentes de saúde permitiram entender o funcionamento das UBS e a logística do trabalho dos médicos, enfermeiros e agentes de saúde. Isto foi essencial também para a delimitação das UBS e escolha da unidade em análise. Foram estabelecidos contatos para ajuda nas abordagens com os usuários.

A pesquisa documental proporcionou coleta de informações e a delimitação e caracterização das UBS. De posse da relação das UBSs da cidade em estudo, as mesmas foram estratificadas quanto à sede: própria ou alugada conforme mostrado no Quadro 2. Foram selecionadas as UBSs de sede própria por terem maior estabilidade quanto ao local de instalação e por entender que proporcionam aos órgãos responsáveis maior possibilidade de adequações no espaço urbano. Dentre estas, foi escolhida a UBS localizada no distrito sede com maior porcentagem de idosos em relação ao número de pessoas cadastradas (Quadro 3). 


\section{Quadro 1 - Variáveis do estudo}

\begin{tabular}{|c|c|c|}
\hline Dimensões & Variáveis & Indicadores \\
\hline \multirow[t]{6}{*}{$\begin{array}{l}\text { Barreiras } \\
\text { Biológicas }\end{array}$} & $\begin{array}{c}\text { Alterações no sistema } \\
\text { músculoesquelético e } \\
\text { equilíbrio }\end{array}$ & $\begin{array}{c}\text { Andarem superfícies irregulares, desviar } \\
\text { de obstáculos, executar ações de } \\
\text { forma rápida, flexibilidade }\end{array}$ \\
\hline & Alterações visuais & $\begin{array}{l}\text { Enxergar com clareza, perceber } \\
\text { desníveis, perceber os limites do } \\
\text { passeio }\end{array}$ \\
\hline & $\begin{array}{l}\text { Alterações no sistema } \\
\text { cardiovascular e pulmonar }\end{array}$ & Caminhar por longos percursos \\
\hline & $\begin{array}{c}\text { Alterações no sistema } \\
\text { auditivo }\end{array}$ & $\begin{array}{c}\text { Perceber falas, entender o que as } \\
\text { pessoas falam em locais com barulho, } \\
\text { ouvir barulho do trânsito }\end{array}$ \\
\hline & Alterações psicológicas & Compreender a sinalização \\
\hline & Uso de medicamentos & Ocorrer confusão mental \\
\hline \multirow[t]{2}{*}{$\begin{array}{l}\text { Características } \\
\text { físicas do } \\
\text { espaço } \\
\text { urbano }\end{array}$} & Condições das calçadas & $\begin{array}{c}\text { Tipo de pavimentação, nivelamento, } \\
\text { obstáculos (degraus, mobiliário } \\
\text { urbano, etc.), largura, declividade e } \\
\text { altura do meio-fio, iluminação }\end{array}$ \\
\hline & Condições das ruas & $\begin{array}{l}\text { Tipo de pavimentação, faixas de } \\
\text { travessia, fluxo de veículos, iluminação }\end{array}$ \\
\hline \multirow[t]{3}{*}{$\begin{array}{l}\text { Características } \\
\text { dos meios de } \\
\text { transporte }\end{array}$} & $\begin{array}{c}\text { Transporte por carro, táxi, } \\
\text { moto, bicicleta, charrete, } \\
\text { cavalo e similares }\end{array}$ & $\begin{array}{c}\text { Condições das vagas de } \\
\text { estacionamento, condições de } \\
\text { transposição da rua para a calçada }\end{array}$ \\
\hline & Transporte por ônibus & $\begin{array}{c}\text { Condições dos abrigos de ônibus, } \\
\text { adequação dos ônibus para Pessoas } \\
\text { com mobilidade reduzida, preparo dos } \\
\text { motoristas e cobradores no } \\
\text { atendimento aos idosos, condições de } \\
\text { transposição da rua para o veículo, } \\
\text { presença de assentos preferenciais }\end{array}$ \\
\hline & Transporte a pé & Envolve os indicadores acima \\
\hline
\end{tabular}

Fonte: Resultados da Pesquisa

\section{Quadro 2 - Relação das UBSs quanto à situação da sede}

\begin{tabular}{|c|c|}
\hline UBS & Situação da Sede \\
\hline Amoras & Alugada \\
\hline Bom Jesus & Alugada \\
\hline Cachoeirinha & Própria \\
\hline João Brás & Alugada \\
\hline Nova Era & Alugada \\
\hline Nova Viçosa / Posses & Própria \\
\hline Novo Silvestre & Própria \\
\hline Santa Clara & Alugada \\
\hline Santo Antônio I & Alugada \\
\hline Santo Antônio II & Alugada \\
\hline São José/Barrinha/Cidade Nova & Própria \\
\hline São José do Triunfo & Própria \\
\hline São Sebastião & Alugada \\
\hline Silvestre & Alugada \\
\hline
\end{tabular}

Fonte: Resultados da Pesquisa 


\section{Quadro 3 - Porcentagem de idosos em relação ao número de pessoas cadastradas por UBS}

\begin{tabular}{|c|c|c|c|}
\hline UBS & $\begin{array}{c}\text { No de } \\
\text { pessoas } \\
\text { cadastradas }\end{array}$ & $\begin{array}{c}\text { Total de } \\
\text { idosos }\end{array}$ & $\begin{array}{c}\text { \% idosos em relação ao no } \\
\text { de pessoas cadastradas }\end{array}$ \\
\hline Cachoeirinha & 1.816 & 278 & 15,31 \\
\hline Nova Viçosa/Posses & 6.006 & 559 & 9,31 \\
\hline $\begin{array}{c}\text { Novo Silvestre } \\
\text { Nosé/Barrinha/Cidade } \\
\text { Nova }\end{array}$ & 1.883 & 248 & 13,17 \\
\hline São José do Triunfo & 4.090 & 546 & 13,35 \\
\hline
\end{tabular}

Fonte: Resultados da Pesquisa

Desta forma, o estudo está sendo realizado na UBS São José/Barrinha/Cidade Nova, localizada na área urbana do município. A UBS está situada na Rua Leonor de Oliveira, no Bairro Cidade Nova. Essa UBS é formada por duas Equipes de Saúde da Família (ESFs), uma composta pelos Bairros São José e Cidade Nova e outra composta pelo Bairro Barrinha e Zona Rural. Estas equipes atuam juntas em 7 micro-áreas que incluem áreas urbanas e rurais. Como o foco da pesquisa é o espaço urbano, serão destacadas na Figura 1 apenas as micro-áreas a ele pertencentes.

\section{Figura 1 - Distribuição da área da abrangência da UBS São José/Barrinha/Cidade Nova, por micro-áreas}

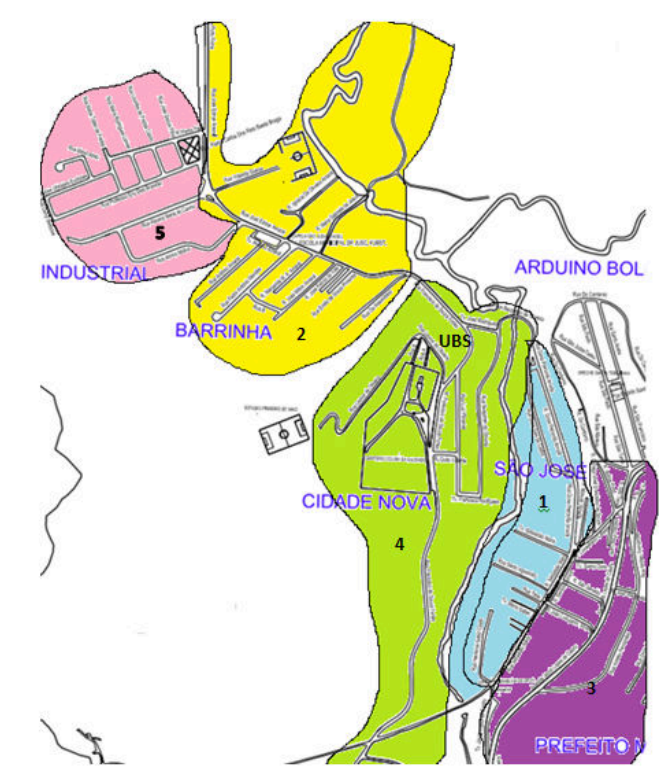

Fonte: Resultados da Pesquisa

Estes resultados contribuirão para a segunda parte da pesquisa que irá caracterizar o espaço urbano contíguo à UBS em estudo quanto às barreiras físicas; identificar as alterações funcionais decorrentes do processo de envelhecimento e correlacioná-los com o uso do espaço; verificar como os 
idosos percebem e se comportam no espaço urbano contíguo à UBS e descrever a mobilidade dos idosos no espaço urbano adjacente à UBS. As próximas etapas da pesquisa serão: aplicação e análise Walkthrough, entrevistas com os idosos ou com seus cuidadores sobre a situação auto referida de saúde, observação comportamental e Walkthrough comentada.

\section{CONSIDERAÇÕES FINAIS}

Esta etapa da pesquisa permitiu o mapeamento das UBSs de Viçosa-MG, a escolha da UBS para investigação e sua respectiva área de abrangência. Possibilitou ainda entender o funcionamento da UBS e a logística do trabalho dos médicos, enfermeiros e agentes de saúde. Os contatos estabelecidos por meio das entrevistas exploratórias não estruturadas serão essenciais para as futuras abordagens aos usuários. Além disso, com a revisão de literatura, foi possível estabelecer as variáveis para o estudo, que serão de grande importância para a segunda etapa da pesquisa.

Com a realização desta primeira etapa foi possível identificar que, apesar de possuir sede própria e de acordo com as especificações legais, a unidade não possui localização adequada devido à sua extensa área de abrangência, necessitando de pontos de apoio para o atendimento aos usuários das localidades rurais. Além disso, o acesso à UBS apresenta barreiras como a BR 356 que torna o trajeto perigoso, pois possui trânsito intenso, curvas fechadas e longos trechos sem acostamentos ou calçadas.

O acesso dos moradores do Bairro São José fica comprometido ainda devido a existência de uma ponte improvisada (pinguela) que compromete a segurança dos mesmos. A via onde a unidade está instalada tem calçamento de pedras fincadas que dificulta o acesso de muitos moradores, principalmente idosos e usuários com dificuldade de locomoção, por serem escorregadias em dias de chuva e por representarem risco de quedas. Sendo assim, o princípio do Primeiro Contato, que diz que a AP deve ser a porta de entrada de fácil acesso aos usuários para o sistema de serviços de saúde, é ferido.

Com este trabalho foi possível reforçar que, na medida em que se tornam necessárias intervenções nas edificações frequentadas pelos idosos, a fim de se reduzir a probabilidade de quedas, é preciso também redirecionar as práticas no que se refere à adequação do espaço urbano, considerado como fundamental para a manutenção da autonomia e independência dos mesmos.

\section{AGRADECIMENTOS}

Ao Grupo de Pesquisa INOVA e à Universidade Federal de Viçosa. 


\section{REFERÊNCIAS BIBLIOGRÁFICAS}

ASSOCIAÇÃO BRASILEIRA DE NORMAS TÉCNICAS (ABNT). NBR 9050: Acessibilidade a edificações, mobiliário, espaços e equipamentos urbanos, 2 ed. Rio de Janeiro, 2004. Disponível em: < http://www.pessoacomdeficiencia. gov.br/app/sites/default/files/arquivos/\%5Bfield_generico_imagens-filefielddescription\%5D_24.pdf>. Acesso em: 10 set. 2014.

ARAUjO, T. C. N., ALVES, M. I. C. Perfil da população idosa no Brasil. Revista Unati, v. 3, n. 3, Rio de Janeiro, fev. 2000. Disponível em: < http://revista.unati. verj.br/scielo.php?script=sci_arttext\&pid=\$1517-59282000000100002\&lng =pt\&nr m=iso>. Acesso em: 13 set. 2013.

BALBINOTTI, H.B. Adulto Maduro: o pulsar da vida, Porto Alegre: WS Editora, 2003.

BARRETO, K. M. L. Envelhecimento, mobilidade urbana e saúde: um estudo da população idosa. 2012. 177 p. Tese ( Doutorado em Saúde Pública) - Centro de Pesquisa Aggeu Magalhães, Fundação Oswaldo Cruz. Recife. 2012. Disponível em: < http://www.cpqam.fiocruz.br/bibpdf/2012barreto-kml.pdf>. Acesso em: 01 set. 2013.

BINS ELY, V. H. M., DORNELES,V. G. Acessibilidade espacial do idoso no espaço livre urbano. In: Congresso Brasileiro de Ergonomia, 4 Fórum Brasileiro de Ergonomia, II Abergo jovem, II Congresso Brasileiro de Iniciação em Ergonomia 2, Anais... Curitiba: ABERGO, 2006. Disponível em: <http://pitagoras.unicamp. br/ teleduc/cursos/diretorio/tmp/2549/portfolio/item/32/abergo-26.pdf>.

Acesso em: 03 set. 2013.

BRASIL. Ministério da Saúde. Política Nacional de Atenção Básica. Brasília: 2012. $110 \mathrm{p}$.

2003.

Ministério da Saúde. Estatuto do Idoso. Brasília. Ministério da Saúde,

CAMBIAGHI, S. Desenho universal: métodos e técnicas para arquitetos e urbanistas. São Paulo: Senac, 2007.

CHILD, S. et al. Factors Influencing the Implementation of Fall-Prevention Programmes: A Systematic Review and Synthesis of Qualitative Studies. Implementation Science, IS 7, 2012, p. 91. Disponível em: < file:///C:/Users/ dell/Desktop/nihms-413570.pdf>. Acesso em: 17 jun. 2015.

CHOI, H., HAYWARD, R. A., LANGA, K. M. Fall Associated Difficulty with Activities of Daily Living (ADL) in Functionally Independent Older Adults Aged 65 to 69 in the United States: A Cohort Study. Journal of the American Geriatrics Society, 2013. Disponível em: < http://www.ncbi.nlm.nih.gov/pmc/articles/PMC 3807864/> . Acesso em: 15 jun. 2015.

CONASS. Conselho Nacional de Secretários de Saúde. Atenção Primária e Promoção da Saúde. Brasília: CONASS, 2011. 197 p.

CUNHA, M. V.P.O., COSTA, A. D. L. Diretrizes projetuais para a acessibilidade física do idoso ao espaço público urbano: a Praça São Gonçalo, João Pessoa - PB. In: 2Simpósio Brasileiro de Qualidade do Projeto no Ambiente Construído $X$ Workshop Brasileiro de Gestão do Processo de Projeto na Construção de Edifícios $X$, Anais... Rio de Janeiro: SBPQ, 2011. Disponível em: 
<http://www.iau.usp.br/ocs/index.php/sbqp2011/sbqp2011/paper/viewFile/283 /193. Acesso em: 10 de out. 2013.

DISCHINGER, M., BINS ELY, V. H. M., PIARDI. S. M. D. G. Promovendo acessibilidade espacial nos edifícios públicos: Programa de Acessibilidade às Pessoas com Deficiência ou Mobilidade Reduzida nas Edificações de Uso. Florianópolis : MPSC, 2012.

GIL, A. C. Métodos e técnicas em pesquisa social. 5 ed. São Paulo: Atlas, 1999.

GUNTHER, H., PINHEIRO, J. Q., GUZZO, R. S. L. Psicologia Ambiental: entendendo as relações do homem com seu ambiente, Campinas: Alínea, 2006.

LIBOREIRO, M. C. et al. Atenção Integral à Terceira Idade em Viçosa, MG pela Parceria UFV e Prefeitura. In: 2 Congresso Brasileiro de Extensão Universitária. Belo Horizonte: 2004. Disponível em: <https://www.ufmg.br/congrext/Saude/ Saude30.pdf >. Acesso em: 15 ago. 2013.

MARCHON, R. M, CORDEIRO, R. C., NAKANO, M. M. Capacidade Funcional: estudo prospectivo em idosos residentes em uma instituição de longa permanência. Revista Brasileira Geriatria e Gerontologia, v.13, n.2, Rio de Janeiro, 2010. Disponível em: < http://revista.unati.uerj.br/scielo.php?script= sci_arttext\&pid=S1809-982320 $10000200005 \&$ Ing=pt\&nrm=iss $>$. Acesso em: 10 ago. 2014.

MARTINS, G. B. Avaliação da capacidade funcional de idosos institucionalizados e não institucionalizados. 2006. 69p. Trabalho de Conclusão de Curso (TCC). Curso de Fisioterapia da Universidade do Sul de Santa Catarina, Tubarão. Disponível em: <http://www.fisio-tb.unisul.br/Tccs/06b/ gilmara/tccgilmara.pdf >. Acesso em: 16 set. 2013.

MESSIAS, M. G., NEVES, R. F. A influência de fatores comportamentais e ambientais domésticos nas quedas em idosos. Revista Brasileira Geriatria e Gerontologia, v.12, n.2, Rio de Janeiro, 2009. Disponível em: < http://www.crdeunati.verj.br/img_tse/v12n2/pdf/art_10.pdf>. Acesso em: 10 ago. 2013.

MINAS GERAIS. Secretaria de Estado de Saúde. Atenção à saúde do idoso. Belo Horizonte: SAS/MG, 2006. 186 p.

NICOLUSSI, A. C. et al. Qualidade de vida em idosos que sofreram quedas: revisão integrativa da literatura. Ciência \& Saúde Coletiva, , v. 17, n. 3, Ribeirão Preto, mar. 2012. Disponível em: < http://www.scielo.br/scielo.php?script= sci_arttext\&pid=S1413-81232012000300019>. Acesso em: 02 ago. 2013.

OKAMOTO, J. Percepção Ambiental e Comportamento: visão holística da percepção Ambiental na Arquitetura e na Comunicação, São Paulo: Mackenzie, 2002.

QUIVY, R., CAMPENHOUDI, L. V. Manual de investigação em ciências sociais. Lisboa: Gradiva, 2005.

TORRES, M. M., SÁ, M. A. A. S. Inclusão social de idosos: um longo caminho a percorrer. Revista Ciências Humanas, v. 1, n. 2, Taubaté, 2008. Disponível em:< http://www.unitau.br/revistahumanas>. Acesso em: 13 ago. 2013.

VERAS, R. Envelhecimento populacional contemporâneo: demandas, desafios e inovações. Revista Saúde Pública, v.43, n.3, São Paulo, mai/jun, 2009. Disponível em: <http://www.scielo.br/pdf/rsp/2009nahead/224.pdf>. Acesso em: 16 ago 2013. 
WOLFF, S. H. (org.). Vivendo e envelhecendo: recorte de práticas sociais nos Núcleos de Vida Saudável. São Leopoldo: Unisinos, 2009. 175p. 